\title{
APPLICATION DE LA MÉTHOde DES ELEMENTS FINIS À LA MOdGLISATION DE STRUCTURES PÉRIODIQUES ACTIVES
}

\author{
A.C. HLADKY-HENNION et J.N. DECARPIGNY \\ Laboratoire d'Acoustique, U.R.A. 253 C.N.R.S., Institut Supérieur d'Electronique du Nord, \\ 41 boulevard Vauban, F-59046 Lille cedex, France
}

\begin{abstract}
Résumé: Un modèle mathématique tridimensionnel a été développé pour analyser le rayonnement $d$ 'une onde acougtique plane par un réseau periodique actif à une ou deux dimensions. Il repose sur la méthode des eléments finis et exploite le code ATILA. Ce papier décrit les résultats obtenus pour différents types de piézocomposites 1-3. La comparaison à des résultats analytiques ou à des mesures permet une excellente validation du modèle.
\end{abstract}

\begin{abstract}
A three-dimensional mathematical model has been developed to analyze the radiation of a plane acoustic wave from simple or doubly periodic active structures. It relies upon the finite element method and uses the AIIIA code. This paper describes results obtained for the radiation of a plane wave from piezocomposite 1-3. Comparison between finite element results and analytical or experimental results demonstrates the accuracy of the model.
\end{abstract}

\section{Introduction}

Dans le domaine de l'acoustique sous-marine et de l'imagerie ultrasonore, certaines structures actives peuvent être décrites, pour permettre i'analyse numérique de leur comportement, comme des structures péxiodiques. Ainsi, les matêriaux piézocomposites 1-3 peuvent être considérếs comme des réseaux bidimensionnels de barrettes parallèles de PZT, ingérées dans une matrice en résine. Ces matériaux, dont la sensibilité aux effets mécaniques transverses est moindre que celle des céramiques, sont dotés d'une excellente sensibilité hydrostatique qui les rend avantageux pour la conception d'hydrophones. De même, les antennes de chasse aux mines ou d'imagerie acoustique sous-marine sont souvent constituées d'un grand nombre de transducteurs et peuvent être considérées comme des réseaux périodiques à une ou deux dimensions, dont 1 'analyse doit permettre de préciser les importants effets d'interaction entre transducteurs voisins. Enfin, l'étude de revêtements anéchoïques actifs ou semi-actifs conçus comme des réseaux d'éléments absorbants peut également relever de la même approche.

L'objet de cette communication est l'étude du cas particulier des piézocomposites 1-3. De nombreux auteurs ont étudié expérimentalement ces matériaux et ont défini l'influence des propriétés de chaque constituant et de la géométrie sur le comportement global du composite $[1,2]$. D'autres auteurs ont établi un modele physique simple, supposant un mode d'épaisseur. Ce modèle est valide lorsque les periodes restent petites vis à vis de l'épaisseur, le composite pouvant alors être traité comme un milieu homogène [3-5]. Mais l'analyse de réseaux complexes du fait de leur géométrie, des matériaux qui les constituent, de la direction de polarisation ou des modes de vibration concernés, nécessite une approche numérique. La méthode des éléments finis, avec l'aide du cođe ATILA, a déjà permis de modéliser les structures périodiques passives à une [6] ou deux dimensions [7]. Elle fait l'objet ici d'une extension aux piézocomposites. La communication rappelle d'abord l'approche théorique 
et montre comment la piézoélectricité peut être prise en compte. Ensuite, elle illustre l'intérêt de la méthode par plusieurs applications au calcul de la sensibilité en émission ou en réception de piézocomposites 1-3.

\section{Formulation théorique}

Le formalisme mathématique nécessaire à la description de structures périodiques est présenté pour un domaine d'étude tridimensionnel, la périodicité étant à deux dimensions (Figure 1). Le domaine est décomposé en trois régions successives par deux plans parallèles au plan de la structure rayonnante, notés $s^{+}$et $s^{-}$. Les première et troisième régions sont des domaines fluides gemi-infinis, dans lesquels le champ de pression est développé en série d'ondes planes progressives et

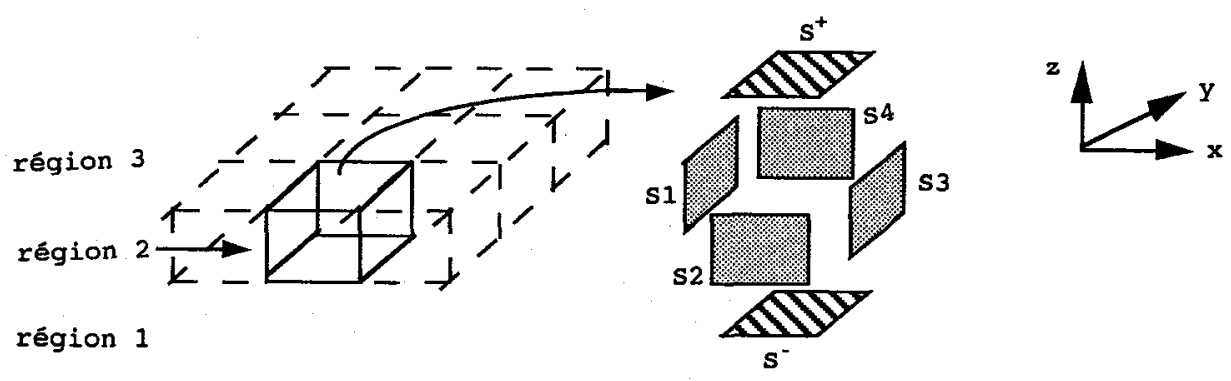

Figure 1: Structure périodique dans deux directions.

évanescentes. Dans la première région, l'une des ondes progressives est l'onde incidente, si elle existe (cas de la reception). La seconde region comprend les structures rayonnantes périodiques et une partie du fluide environnant. Dans cette région, la double périodicité du problème est prise en compte à l'aide de conditions cycliques du type Bloch-Floquet, c'est à dire d'une relation de phase spécifique entre des points distants d'un nombre entier de fois le pas du réseau. L'étude de cette région peut ainsi être réduite à une maille élémentaire. Celle-ci fait l'objet d'un maillage par eléments finis, décrivant les domaines fluide, solide et piézoélectrique [8]. Les relations de Bloch-Floquet, appliquées aux valeurs du déplacement, de la pression ou du potentiel electrique sur des noeuds séparés d'une période, sont utilisées pour définir les conditions aux limites entre des cellules élémentaires voisines, sur les plans s1, s2, s3 et $\mathrm{s} 4$. Ces relations permettent des combinaisons linéaires de certaines lignes et colonnes du système, i.e. la condensation des degrés de liberté appartenant aux faces $s 3$ et 54 limitant la cellule élémentaire. Par ailleurs, les equations de continuité pour le champ de pression et sa dérivée normale sont écrites sur la frontière entre les régions 1 et 2 d'une part, 2 et 3 d'autre part. La prise en compte de l'effet des domaines fluides externes introduit des relations matricielles entre les valeurs nodales de la dérivée normale de la pression et les valeurs nodales de la pression. Classiquement, les équations du système fournissent les champs de déplacement, de pression et du potentiel électrique. Selon le mode de connexion du domaine piézoélectrique, les quantités calculées sont la sensibilité en réception ou la réponse par volt du transducteur. Lorsque la structure est infinie dans une direction, le problème est bidimensionnel et des simplifications évidentes sont réalisées [6].

\section{Résultats}

II.1. Modélisation d'un piézocomposite 1-3 et validation

Il s'agit d'étudier le rayonnement d'un piézocomposite 1-3, noté échantillon 200 dans les références $[1,2]$, correspondant à 208 de PzT5A noyé dans de l'époxy. La face arrière du transducteur est dans l'air. La figure 2 présente la sensibilité en réception en fonction de la fréquence. L'accord entre résultats numériques et expérimentaux est bon. Aux fréquences inférieures à celle du maximum principal, les minima sur la courbe correspondent aux modes latéraux, associés à des ondes 
stationnaires dans l'époxy et se propageant dans la direction orthogonale à l'axe des barrettes de PZT. Les fréquences de ces modes sont relativement indépendantes de l'épaisseur du composite et sont reliées à la périodicité latérale des barrettes. Le maximum principal correspond, lui, à l'excitation du mode d'épaisseur des barrettes, qui agissent de plus comme des sources d'ondes transverses se propageant dans 1'époxy. La figure 3 présente les variations avec la fréquence de la réponse par volt, mesurée et calculée par eléments finis. Elle montre un bon accord.
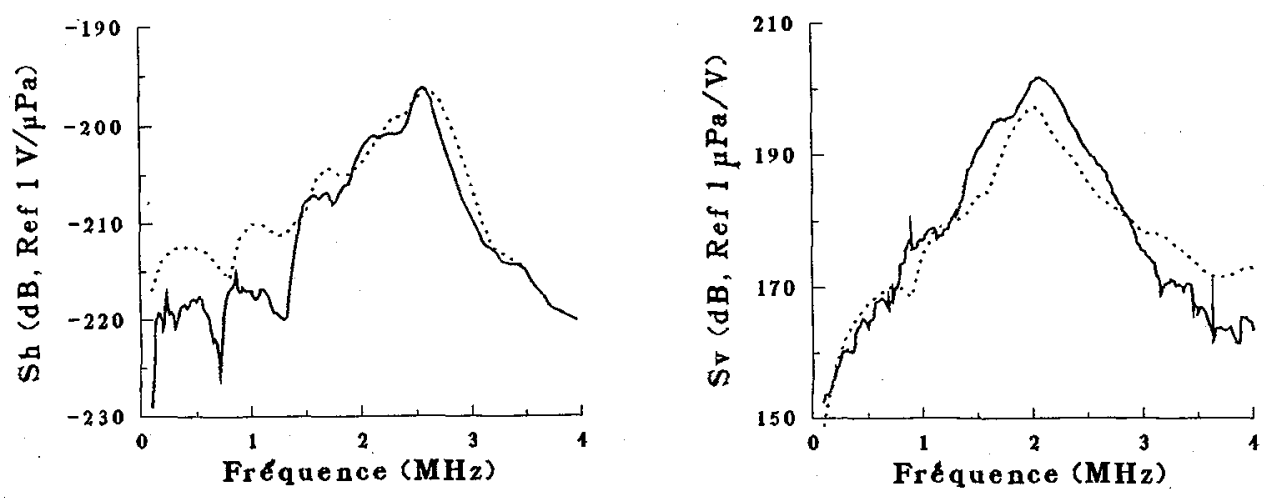

Piézocomposite 1-3: Fiqure 2: Sensibilité en réception; Figure 3: Réponse par volt Traits pleins: mesures, traits pointillés: résultats éléments finis.

II.2. Etude paramétrique d'un piézocomposite 1-3

En vue d'étudier les propriétés des piézocomposites 1-3, un modèle physique simple a déjà été exploité [3-5]. Dans ce modèle, le composite est traité comme un milieu homogène, avec des propriétés effectives, ce qui convient si les dimensions latérales des barrettes et leurs espacements sont suffisamment petits par rapport à l'épaisseur. Plusieurs échantillons, bordés de part et d'autre par de l'eau, sont modélisés ci-après dans le but d'étudier les limites de validité de la méthode d'homogénéisation, en fonction du rapport de l'épaisseur h a la périodicité 2d. La périodicité spatiale est supposée égale à $500 \mu \mathrm{m}$, avec des barrettes de section carrée, de $250 \mu \mathrm{m}$ de côté, correspondant à $25 \%$ de PZT. Les barrettes sont enrobées d'époxy. La figure 4 présente les variations avec la fréquence de la sensibilité en réception obtenues analytiquement et par éléments finis, lorsque le rapport h/2d est égal à $0.8,3$ et 6 . Au delà de 6 , le modèle analytique simple décrit correctement le comportement du composite. Dans ce cas, les fréquences des modes latéraux sont élevées et le mode d'épaisseur est prédominant dans la bande utile.
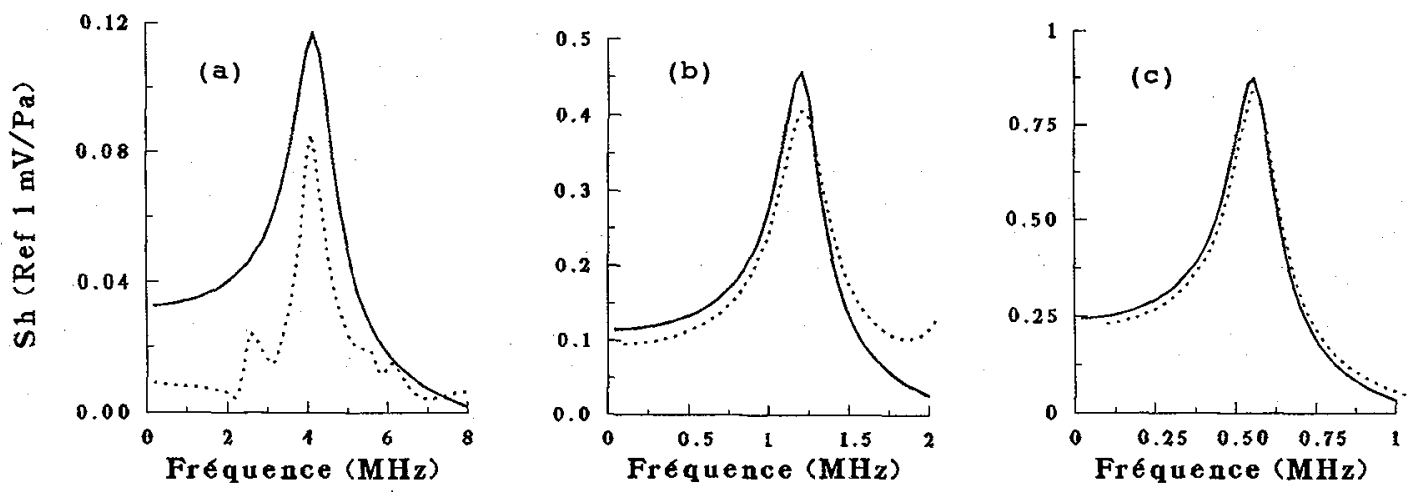

Fiquxe 4: Piézocomposite 1-3: sensibilité en réception en fonction du rapport h/2d. a) $: \mathrm{h} / 2 \mathrm{~d}=0.8 ; \mathrm{b}): \mathrm{h} / 2 \mathrm{~d}=3 ; \mathrm{c}): \mathrm{h} / 2 \mathrm{~d}=6$

Traits pleins: résultats analytiques, traits pointillés: résultats élêments finis. 
Ensuite, utilisant l'echantilion pour lequel $\mathrm{h} / 2 \mathrm{~d}=6$, d'autres echantillons sont etudies, avec différents pourcentages volumiques de PZT: 48, 468 et 908. La figure 5 présente les variations avec la fréquence de la réponse par volt. L'accord est bon mais la valeur du maximum differe, probablement parce que le champ de déplacement dans l'époxy n'est pas réellement uniforme.
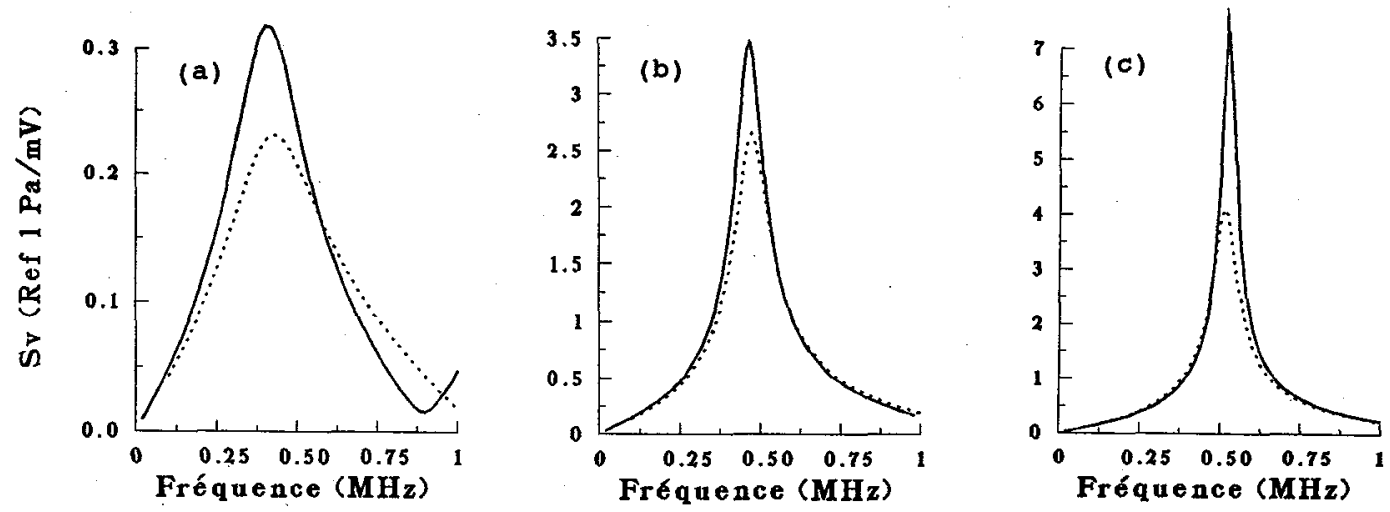

Fiqure 5: Piézocomposite 1-3: réponse par volt en fonction du pourcentage de PzT.

a): 4 \& (b): 46 \& ; c): 908

Traits pleins: résultats analytiques, traits pointillés: résultats éléments finis.

\section{Conclusion}

L'analyse du comportement acoustique de réseaux actifs à périodicité simple ou double a été conduite à l'aide du modẻle éléments finis, avec le code ATILA. Un bon accord a eté trouvé entre les resultats numériques et les mesures. Leg limites d'un modèle analytique d'homogéneisation ont de plus eté montrées. I'un de nos objectifs est maintenant d'étudier des matériaux composites ayant un autre type de connectivite, par exemple les composites 1-3-0 [9]. De même, la methode est étendue à la modélisation de structures à periodicité tridimensionnelle. Elle permet ainsi de déterminer, par exemple, les propriétés homogénéisées de ces matériaux, dans la limite des grandes longueurs d'onde.

\section{Références}

[1]T.R. GURURAJA, W.A. SCHULZE, L.E. CROSS, R.E. NEWWHAM, B.A. AULD, IEEE Trans. Sonics and Ultras., SU 32, 481-498.

[2]T.R. GURURAJA, W.A. SCHULZE, L.E. CROSS, R.E. NEWNHAM, B.A. AULD, IEEE Trans. Sonics and Ultras., sU 32, 499-513.

[3]W.A. SMITH, A.A. SHAULOV, B.M. SINGER, Ultrasonics SYmp., 539-544 (1984).

[4]W.A. SMITH, B.A. AULD, IEEE Trans. Sonics and Ultras., 38, 40-47, (1990).

[5]G. HAYWARD, J.A. HOSSACK, J. Acoust. SOC. Am, 88, 599-608, (1990).

[6]A.C. HENNION, R. BOSSUT, J.N. DECARPIGNY, C. AUDOLY, J. Acoust. Soc. Am., 87, 1861-1870. (1990).

[7]A.C. HLADKY-HENNION, J.N. DECARPIGNY, à parẫtre dans J. Acoust. Soc. Am., (1991).

[8]0.C. ZIENKIEWICZ, "The finite element method", Ed. Mac Graw Hill, (1977).

[9]M.J.HAUN, R.E NEWNHAM, Ferroelectrics, 68, 123-139, (1986). 